\title{
Classical and quantum anisotropic Heisenberg antiferromagnets
}

\author{
W. Selke ${ }^{1}$, G. Bannasch ${ }^{2}$, M. Holtschneider ${ }^{1}$, I.P. McCulloch ${ }^{3}$, D. Peters ${ }^{1}$, S. Wessel ${ }^{4}$ \\ ${ }^{1}$ RWTH Aachen University and JARA-SIM, Department of Theoretical Physics, 52056 Aachen, Germany \\ 2 MPI für Physik komplexer Systeme, 01187 Dresden, Germany \\ 3 University of Queensland, Brisbane, QLD 4072, Australia \\ 4 University of Stuttgart, Institute for Theoretical Physics III, 70550 Stuttgart, Germany
}

Received June 12, 2009, in final form June 30, 2009

\begin{abstract}
We study classical and quantum Heisenberg antiferromagnets with exchange anisotropy of XXZ-type and crystal field single-ion terms of quadratic and quartic form in a field. The magnets display a variety of phases, including the spin-flop (or, in the quantum case, spin-liquid) and biconical (corresponding, in the quantum lattice gas description, to supersolid) phases. Applying ground-state considerations, Monte Carlo and density matrix renormalization group methods, the impact of quantum effects and lattice dimension is analysed. Interesting critical and multicritical behaviour may occur at quantum and thermal phase transitions.
\end{abstract}

Key words: Heisenberg antiferromagnets, Monte Carlo simulation, DMRG, biconical phase, supersolid phase, multicritical point

PACS: $05.10 . \mathrm{Ln}, 75.10 . \mathrm{Jm}, 75.40 . \mathrm{Mg}, 75.40 . \mathrm{Cx}$

\section{Introduction}

Uniaxially anisotropic Heisenberg antiferromagnets in a magnetic field along the easy axis have been studied quite extensively in the past, both experimentally and theoretically $[1,2]$. Typically, in the ground state and at low temperatures, they display the antiferromagnetic (AF) and, when increasing the field, the spin-flop (SF) phase, as depicted in figure 1.

(a)

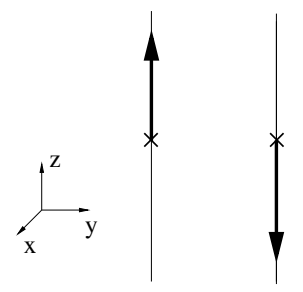

(b)

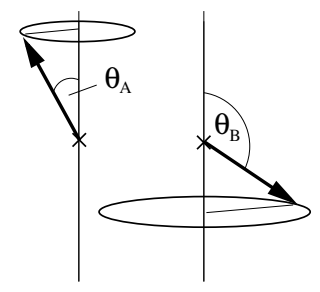

(c)

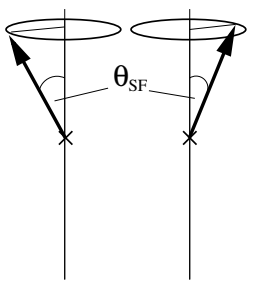

Figure 1. Spin orientations on neighboring sites showing perfect antiferromagnetic (a), biconical (b), and spin-flop (c) structures.

There may also occur intermediate or mixed spin structures of 'biconical' [3] (BC) type. They are characterized by distinct turning angles, $\Theta_{A}$ and $\Theta_{B}$, with respect to the easy axis for neighboring spins situated on the two sublattices, see also figure 1. Quite a few experiments related to BC structures have been performed over the years $[4,5]$.

Recent studies deal with the influence of defects $[6,7]$, of the lattice dimension, of subtleties of the anisotropic interactions, as well as of quantum effects on the phase diagrams. In particular, topological and (multi)critical properties of the phase diagrams have been elucidated.

The aim of the present contribution is to draw attention to recent analyses on the prototypical XXZ Heisenberg antiferromagnet in a field and variants. The XXZ model is assumed to have a 
uniaxial exchange anisotropy, with the magnetic field being along the easy axis. Biconical structures may be stabilized by adding crystal-field single-ion terms of quadratic or quartic form and favoring non-uniaxial spin orderings. Much actual interest in these BC structures stems from the fact that they correspond to supersolid structures, when transcribing the Heisenberg antiferromagnet to a quantum lattice gas [8-11]. We shall consider both classical models, with spinvectors of length unity, and quantum models, with spin $S=1 / 2$ and $S=1$. In addition, to discuss the role of lattice dimension, the results for chains, square lattices, and cubic lattices will be presented.

\section{2. $X X Z$ antiferromagnets}

As a starting point of theoretical studies on uniaxially anisotropic Heisenberg antiferromagnets, one often considers the prototypical XXZ model, with the Hamiltonian

$$
\mathcal{H}_{\mathrm{XXZ}}=J \sum_{i, j}\left[\Delta\left(S_{i}^{x} S_{j}^{x}+S_{i}^{y} S_{j}^{y}\right)+S_{i}^{z} S_{j}^{z}\right]-H \sum_{i} S_{i}^{z}
$$

where $J>0$ is the antiferromagnetic exchange coupling between spins being located on neighboring lattice sites $i$ and $j . \Delta$ is the exchange anisotropy, $1>\Delta>0$ in the case of uniaxiality, and $H$ is the applied magnetic field along the easy axis, the $z$-axis.

In what follows, we shall discuss the phase diagrams of the XXZ antiferromagnets for square and cubic lattices, showing both similar and quite distinct features. The phase diagrams have been obtained using mainly Monte Carlo (MC) simulations applying the Metropolis method for classical models and applying stochastic series expansion techniques for quantum systems, augmented by finite-size analyses.

In our MC simulations we studied two-dimensional systems with up to $240^{2}$ classical and up to $150^{2}$ quantum, $\mathrm{S}=1 / 2$, spins. In three dimensions, we simulated the systems with up to $32^{3}$ classical spins. To estimate error bars, we averaged over several rather long runs. In all cases, full periodic boundary conditions have been employed.

\subsection{Square lattice}

The classical XXZ model on a square lattice is known to display in the (temperature T, field $\mathrm{H}$ )-plane ordered AF and SF phases [12-15], see figure 2. The transition to the paramagnetic phase belongs to the Ising universality class along the boundary line of the AF phase, and to the XY (or Kosterlitz-Thouless) universality class along the boundary line of the SF phase.

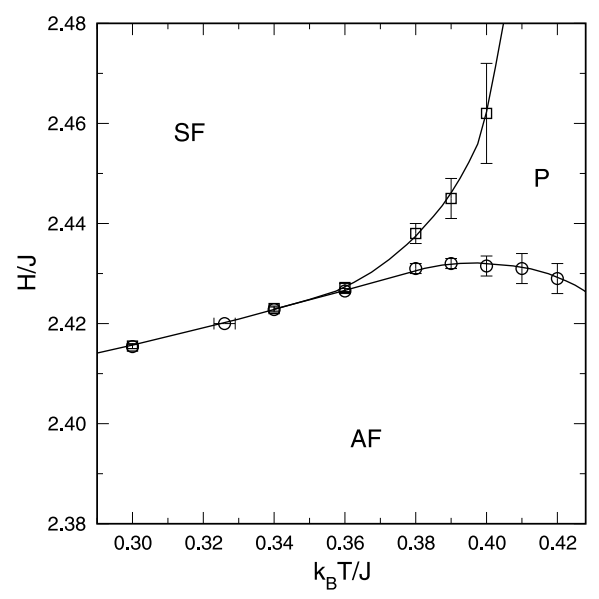

Figure 2. Phase diagram of the classical XXZ model on a square lattice near the maximum of the boundary line of the AF phase, $\Delta=4 / 5$, as obtained using Monte Carlo simulations. 
Extensive Monte Carlo simulations augmented by finite-size analyses [13-15] suggest that there is no direct transition between the AF and SF phases, albeit the two ordered phases approach each other very closely at low temperatures, see figure 2. The intervening, extremely narrow paramagnetic $(\mathrm{P})$ phase and the two ordered phases arise from the highly degenerate ground state occurring at the field $H_{\mathrm{c}}=4 J \sqrt{1-\Delta^{2}}$. At that point, not only $\mathrm{AF}$ and $\mathrm{SF}$ configurations have the same energy, but also biconical structures. The BC structures, figure 1(b), may be described by the tilt angles $\Theta_{A}$ and $\Theta_{\mathrm{B}}$, being uniquely interrelated by [15]

$$
\Theta_{\mathrm{B}}=\arccos \left(\frac{\sqrt{1-\Delta^{2}}-\cos \Theta_{A}}{1-\sqrt{1-\Delta^{2}} \cos \Theta_{A}}\right),
$$

where the tilt angle $\Theta_{A}$ ranges from 0 to $\pi$. Indeed, the degenerate BC configurations have been argued to lead to the intervening disordered phase between the AF and SF phases [15]. Their presence at low temperatures may be conveniently demonstrated by monitoring the probability $p_{2}\left(\Theta_{A}, \Theta_{\mathrm{B}}\right)$ to find the two tilt angles at neighboring sites, as illustrated in figure 3. Similar observations hold for the anisotropic XY antiferromagnet on a square lattice [16].

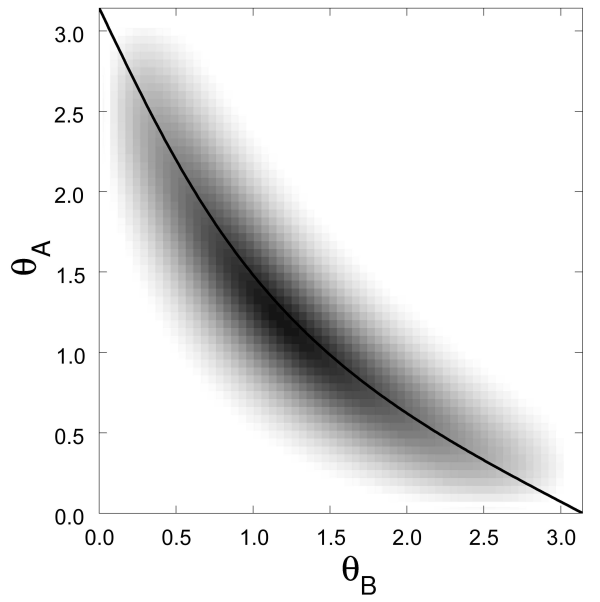

Figure 3. Probability $p_{2}\left(\Theta_{A}, \Theta_{\mathrm{B}}\right)$ showing the correlations between the tilt angles $\Theta_{A}$ and $\Theta_{\mathrm{B}}$ on neighboring sites for the XXZ antiferromagnet on a square lattice, with $80 \times$ 80 spins, at $H / J=2.41, k_{\mathrm{B}} T / J=0.255$, and $\Delta=\frac{4}{5} \cdot p_{2}$ is proportional to the gray scale. The superimposed black line depicts the relation between the two angles $\Theta_{A}$ and $\Theta_{\mathrm{B}}$ in the ground state, equation (2).

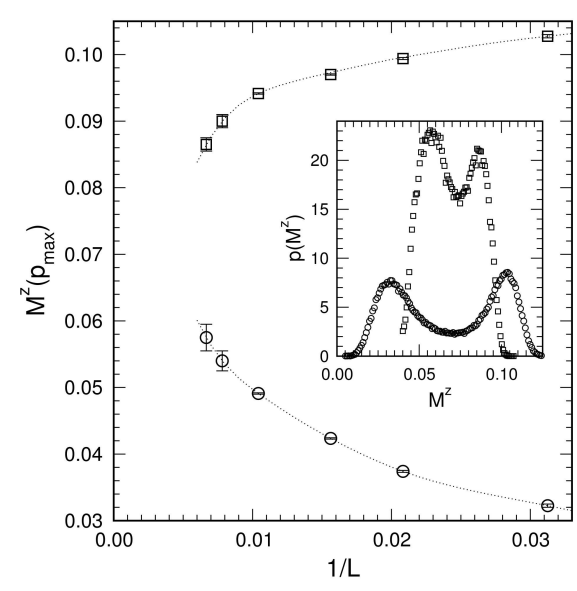

Figure 4. Positions of the maxima of the magnetization histograms as a function of the inverse system size, $1 / L$, for the $\mathrm{S}=1 / 2 \mathrm{XXZ}$ antiferromagnet on a square lattice, with $\Delta=$ $2 / 3$. The inset exemplifies two histograms for systems of linear size $L=32$ (circles) and $L=150$ (squares) at $k_{\mathrm{B}} T / J=0.13$ and the coexistence fields $H / J=1.23075$ and $H / J=$ 1.232245 [15].

The degenerate point at $\left(T=0, H_{\mathrm{c}}\right)$ may be coined a 'hidden tetracritical point' [15,17].

Due to the degeneracy, various physical quantities take at $T=0$ and $H_{\mathrm{c}}$ nontrivial values, depending continuously on $\Delta$. For instance, the magnetization per site is approximately 0.22 for $\Delta=0.8[16]$.

In contrast, the quantum XXZ antiferromagnet with $\mathrm{S}=1 / 2$ on a square lattice seems to exhibit a direct transition of the first order between the AF and SF phases [18]. The triple point, at which the $\mathrm{AF}, \mathrm{SF}$ and $\mathrm{P}$ phases join, has been argued to be a critical endpoint, with the boundary line between the $\mathrm{AF}$ and $\mathrm{P}$ phases being of the first order close to that triple point [18]. The evidence had been provided mainly by quantum Monte Carlo simulations. We did quantum Monte Carlo simulations as well, for the same anisotropy, namely $\Delta=2 / 3$, using stochastic series expansions with directed loop updates [19], enlargening the system sizes and improving the statistics of the 
simulations in comparison with the previous Monte Carlo study [15,18]. Our results suggest that the location of that special point may be shifted towards lower temperatures, compared to the previous estimate [15]. This is illustrated in figure 4, where very accurate MC data for various system sizes are shown at a temperature closely above that of the proposed critical endpoint, where the previous study suggested a transition of the first order between the AF and $\mathrm{P}$ phases. Monitoring the size dependence of the peak positions in the magnetization histograms, figure 4, the two peaks, may well coincide in the thermodynamic limit, signaling a continuous transition [15]. Because we do not find any evidence for BC structures in our simulations of the quantum model, there seems to be no mechanism for destroying long-range order at low temperatures in the quantum case. Thence the direct transition between the AF and SF phases seems to be possible, in accordance with recent quantum MC findings [20]. In general, quantum fluctuations may substantially reduce BC structures, compared to the classical case, as will be discussed below.

\subsection{Cubic lattice}

The classical XXZ antiferromagnet on the cubic lattice displays both ordered AF and SF phases, see figure 5. In agreement with previous simulations [21], we find the transition between the AF and $\mathrm{SF}$ phases to be of the first order [22]. There is again a highly degenerate ground state, now at $H_{\mathrm{c}}=6 J \sqrt{1-\Delta^{2}}$, with BC configurations as described by equation (2). Indeed, by monitoring the probability $p_{2}\left(\Theta_{A}, \Theta_{\mathrm{B}}\right)$, BC configurations are observed to contribute to the thermal fluctuations at low temperatures. But, close to the transition between the AF and SF phases, $p_{2}$ shows pronounced local maxima corresponding to these two phases, indicating phase coexistence at a transition of the first order. In addition, the analysis of other standard thermodynamic quantities gives evidence for a transition of the first order [22].

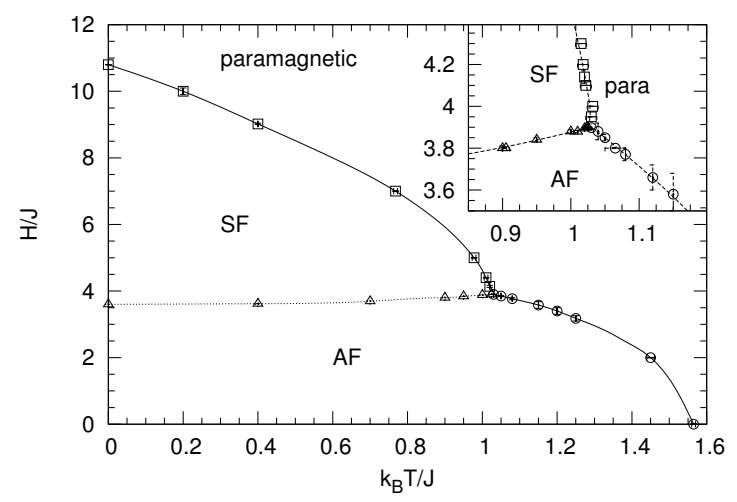

Figure 5. Phase diagram of the XXZ antiferromagnet on the cubic lattice, with $\Delta=0.8$. Inset: Vicinity of the triple point.

The nature of the triple point, at which the AF, SF, and $\mathrm{P}$ phases meet, has been subject to recent renormalization group (RNG) analyses, yielding either some kind of critical endpoint [23] or, possibly depending on the degree of anisotropy $\Delta$, a bicritical point [24]. The latter variant had been suggested in early RNG studies [3] as well.

From our MC simulations [22] we can locate the triple point accurately at $k_{\mathrm{B}} T / J=1.025 \pm 0.015$ and $H / J=3.90 \pm 0.03$, improving the previous estimate [21]. We observe no evidence for a firstorder transition at the SF-P or AF-P boundary lines in the vicinity of the triple point at $\Delta=0.8$ [22], being compatible with the existence of a bicritical point. In fact, we find critical exponents, for instance for staggered longitudinal (i. e. along the easy axis) and transversal susceptibilities, at the AF-P boundary line to belong to the Ising universality class, while those at the SF-P boundary line are clearly consistent with the XY universality class. Perhaps, Monte Carlo studies at different values of the exchange anisotropy $\Delta$ may provide further insights into clarifying the predictions of the RNG treatments. 


\section{Adding single-ion anisotropies}

There is no thermally stable biconical phase in the classical XXZ antiferromagnet. BC configurations occur in the ground state only at the special field, $H_{\mathrm{c}}$, separating AF and SF structures. $\mathrm{BC}$ fluctuations seem to lead to a narrow disordered phase between the AF and SF phases in two dimensions. In three (and, presumably, higher) dimensions they do not destroy the phase transition of the first order between the AF and SF phases, as predicted by mean-field theory.

However, BC structures may be stabilized over a wide range of fields, when introducing additional quadratic or cubic single-ion anisotropies, for example, in the XXZ antiferromagnet favoring non-uniaxial spin orientations. In what follows we shall first consider the phase diagrams, in the $(T$, $H)$ plane, for classical Heisenberg antiferromagnets with quadratic anisotropies on square lattices and with cubic anisotropies on cubic lattices. Finally, we shall present the results on the ground state phase diagram of a quantum, $S=1$, Heisenberg chain with uniaxial exchange anisotropy plus a quadratic single-ion anisotropy in a field.

We use straightforward ground state considerations and Monte Carlo techniques for the classical models. The quantum spin chain at zero temperature is analysed by applying density matrix renormalization group (DMRG) methods. Whenever feasible, the finite-size behavior is taken into account.

In our MC simulations we study the systems with up to $240^{2}$ spins in two dimensions, and with up to $32^{2}$ spins in three dimensions. Details on the ground state DMRG calculations for the $S=1$ quantum Heisenberg chain will be given below.

\subsection{Classical antiferromagnets in two and three dimensions}

In the case of the square lattice, we add to the XXZ model, equation (1), a single-ion term of the quadratic form

$$
\mathcal{H}_{D}=D \sum_{i}\left(S_{i}^{z}\right)^{2}
$$

where the single-ion anisotropy may, depending on the sign of $D$, enhance the uniaxial exchange anisotropy $\Delta(0<\Delta<1)$, when $D<0$, or it may introduce a competing planar anisotropy, $D>0$. The full Hamiltonian is then $\mathcal{H}=\mathcal{H}_{\mathrm{XxZ}}+\mathcal{H}_{D}$.

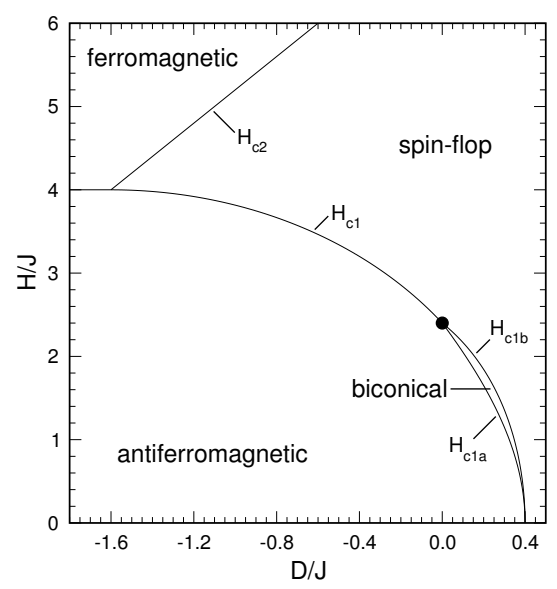

Figure 6. Ground state phase diagram of the classical Heisenberg model with exchange, $\Delta$, and quadratice single-ion, $D$, anisotropy on a square lattice, for $\Delta=0.8$. The filled circle denotes the highly degenerate point at $D=0$ and $H=2.4 J$.

From a straighforward analysis of the ground states one may obtain the phase diagram at zero temperature $[9,16,25]$. An example is shown in figure 6 . There the exchange anisotropy has been fixed to be $\Delta=0.8$. The resulting ground state structures are of AF, SF or BC type. Of course, at 
sufficiently large fields, all spins will eventually point in the direction of the field, i. e. one encounters the ferromagnetic spin configuration, giving rise to the $\mathrm{P}$ phase at non-zero temperatures. As for the XXZ antiferromagnet, the xy-components of the spins order antiferromagnetically in the SF and $\mathrm{BC}$ configurations, as depicted in figure 1, having rotational symmetry.

The most interesting feature of the ground state phase diagram is the region of stable $\mathrm{BC}$ structures, evolving, at $D>0$, from the highly degenerate point of the XXZ model. In that region, the degeneracy is lifted, and the tilt angles $\Theta_{A}$ and $\Theta_{\mathrm{B}}$, being still uniquely interrelated, change continuously with the field $H[16,25]$. Above a critical positive value of $D, D / J>2-2 \Delta$, at the vanishing field, the spins are oriented perpendicular to the easy axis. Applying then a field, a SF structure will be stable, as shown in figure 6 .

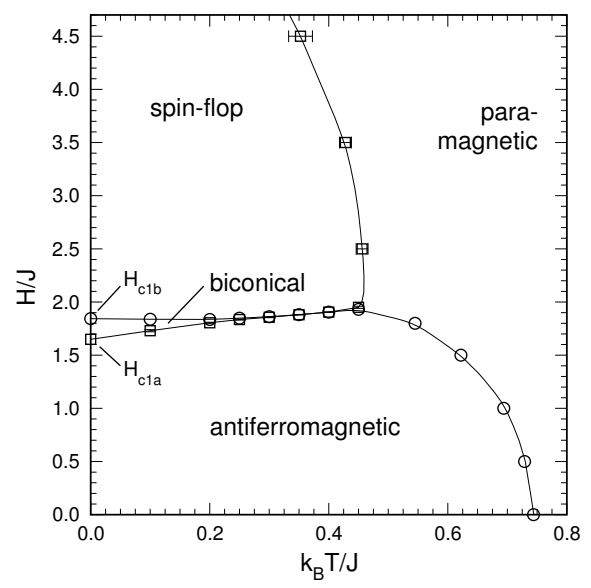

Figure 7. Phase diagram of the XXZ antiferromagnet on a square lattice with a competing single-ion anisotropy, $\Delta=0.8$ and $D / J=0.2$.

At $D>0$, the BC ground states give rise to a stable biconical phase in between the AF and SF phases, as exemplified in figure 7 , setting $D / J=0.2$. In the $\mathrm{BC}$ phase, the order parameters of the $\mathrm{AF}$ and $\mathrm{SF}$ phases, the staggered longitudinal and transversal magnetizations, do not vanish $[1,9$, 10,25]. The BC-AF transition is expected to belong to the XY (or Kosterlitz-Thouless) universality class, while at the BC-SF transition the order parameter of the AF phase vanishes in an Ising-like manner.

In the BC phase, the dominant tilt angles $\Theta_{A}$ and $\Theta_{\mathrm{B}}$ change continuously with the field, when fixing the temperature. That behavior may be inferred from the ground state properties. This has been confirmed in simulations by monitoring the probability $p(\Theta)$ of encountering a spin with the tilt angle $\Theta$ at an arbitrary lattice site, showing two pronounced peaks corresponding to the values of $\Theta_{A}$ and $\Theta_{\mathrm{B}}$ at given temperature and field in the BC phase [25].

It seems an open issue whether the $\mathrm{AF}, \mathrm{SF}, \mathrm{BC}$, and $\mathrm{P}$ phases meet at one point, presumably a tetracritical point [26]. At present, we can locate it, e.g. for the case depicted in figure 7, only with a rather large uncertainty $[16,25]$.

At $D<0$, BC structures are squeezed out by the single-ion term in the ground state. At low temperatures, there seems to be a direct transition of the first order between the AF and SF phases $[16,25]$.

In the case of the cubic lattice, we add to the XXZ model, equation (1), a cubic single-ion anisotropy of quartic form [22]

$$
\mathcal{H}_{\mathrm{CA}}=F \sum_{i}\left[\left(S_{i}^{x}\right)^{4}+\left(S_{i}^{y}\right)^{4}+\left(S_{i}^{z}\right)^{4}\right]
$$

where $F$ denotes the strength of the cubic anisotropy. The sign of $F$ determines whether the spins tend to align along the cubic axes, for $F<0$, case 1 , or, for $F>0$, case 2 , in the diagonal directions of the lattice. Because of these tendencies, the BC, (i. e. BC1 or BC2), structures, as 
well as the SF structures, show no full rotational invariance in the $x y$-plane, in contrast to the XXZ antiferromagnet, with or without quadratic single-ion term. Now, as sketched in figure 8, the spin projections in the $x y$-plane favor four directions [22].

(a)

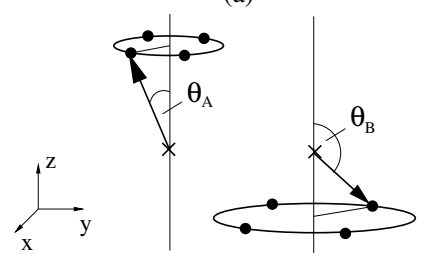

(b)

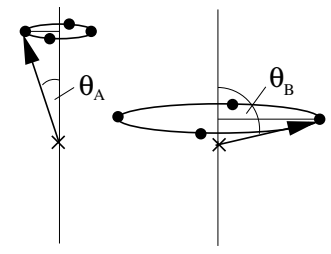

Figure 8. Sketch of discretized biconical structures for the Heisenberg antiferromagnet with cubic anisotropy, in the case of (a) $F>0, \mathrm{BC} 2$, and (b) $F<0$, BC1.

The resulting ground state phase diagram of the full Hamiltonian, $\mathcal{H}=\mathcal{H}_{\mathrm{XxZ}}+\mathcal{H}_{\mathrm{CA}}$, with fixed exchange anisotropy, $\Delta=0.8$, and varying cubic term, $F$, may be easily determined numerically $[22,27]$, as depicted in figure 9 .

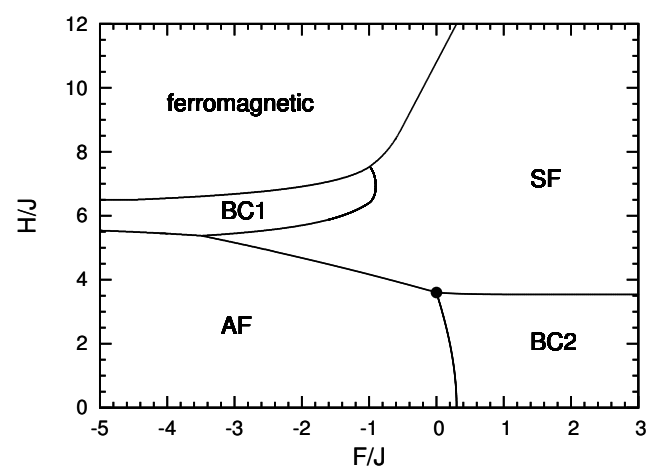

Figure 9. Ground states of the threedimensional classical XXZ antiferromagnet with exchange anisotropy, $\Delta=0.8$, and varying the cubic term $F$.

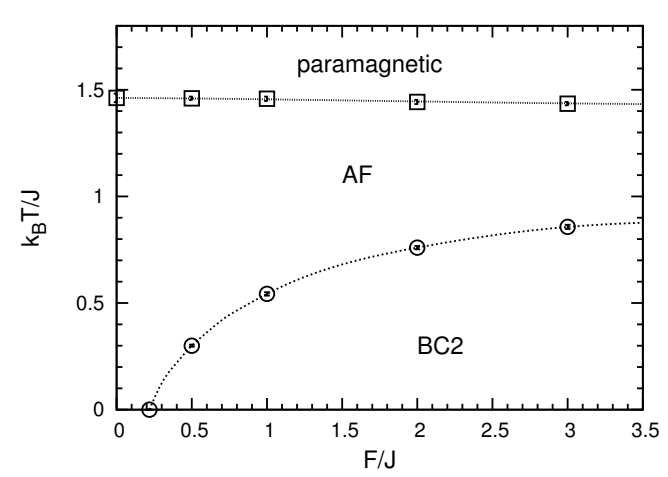

Figure 10. Phase diagram of the XXZ antiferromagnet, $\Delta=0.8$, on a cubic lattice with a positive cubic anisotropy $F$ at fixed field, $H / J$ $=1.8$.

For $F<0$, the transitions to the BC1 structures are typically of the first order, with a jump in the tilt angles, $\Theta$, with respect to the $z$-axis, characterizing the $\mathrm{BC}$ configurations. However, in the reentrance region between the $\mathrm{SF}$ and $\mathrm{BC} 1$ structures at $F / J$ close to -1 , the change in the tilt angles seems to be smooth [22].

Obviously, at non-zero temperatures, several interesting scenarios leading, possibly, to a multicritical behavior, where $\mathrm{AF}, \mathrm{SF}, \mathrm{BC}$, and $\mathrm{P}$ phases meet, may exist. So far, we focussed our attention on two cases [22]:

(i) Positive cubic anisotropy $F>0$, at constant field $H / J=1.8[22]$, see figures 9 and 10 . At small values of $F$, there is an AF ordering at low temperatures. Above the critical value, $F_{\mathrm{c}}=0.218 \ldots J$, the low-temperature phase is of $\mathrm{BC} 2$ type, followed by the AF and $\mathrm{P}$ phases, when increasing the temperature. The transition between the $\mathrm{AF}$ and $\mathrm{P}$ phases is found to belong to the Ising universality class, while the transition between the $\mathrm{BC} 2$ and $\mathrm{AF}$ phases seems to belong to the XY universality class, with the cubic term being then an irrelevant perturbation $[22,28]$.

(ii) Negative $F$, fixing the cubic term, $F / J=-2$, and varying the field, see figure 9 . In accordance with the ground state analysis, we observe, at sufficiently low temperatures, AF, SF, $\mathrm{BC} 1$, and $\mathrm{P}$ phases when increasing the field. Interestingly, the $\mathrm{BC} 1$ phase seems to become unstable when raising the temperature, with the other phases being still present [22]. This may suggest that the three boundary lines between the $\mathrm{BC} 1-\mathrm{P}, \mathrm{SF}-\mathrm{BC} 1$, and $\mathrm{SF}-\mathrm{P}$ phases meet at a multicritical 
point. Of course, further clarification and a search for other, possibly multicritical scenarios at different strengths of the cubic term, $F$, are encouraged.

\subsection{Ground state phase diagrams of $S=1$ anisotropic Heisenberg chains}

In what follows, we shall consider the spin-1 anisotropic antiferromagnetic Heisenberg chain with a quadratic single-ion term in a field described by the Hamiltonian

$$
\mathcal{H}=\sum_{i}\left(J\left(S_{i}^{x} S_{i+1}^{x}+S_{i}^{y} S_{i+1}^{y}+\Delta S_{i}^{z} S_{i+1}^{z}\right)+D\left(S_{i}^{z}\right)^{2}-B S_{i}^{z}\right),
$$

where $i$ denotes the lattice sites. The antiferromagnetic exchange coupling $J$ is again positive. For $\Delta>1$, there is an uniaxial exchange anisotropy, along the direction of the field, $B>0$, the $z$-axis. Note the slight change in defining the exchange anisotropy $\Delta$ compared to that for the XXZ model as given in equation (1). Here we follow the standard notation of previous studies on closely related quantum spin chains [29-31]. For the same reason, the field is now denoted by $B$. Depending on the sign of $D$, the single-ion term leads to a competing planar anisotropy, $D>0$, or to an enhancing uniaxial anisotropy, $D<0$, as already stated above. We shall analyse the ground state properties using DMRG techniques [32,33] for the chains with open boundary conditions and up to $L=128$ sites. In addition, we determine the ground states of corresponding infinite chains with classical spin vectors of length one $[9,25]$.

We shall compare our findings on the quantum spin chain to the ground state properties of the corresponding classical chains, equation (5). To characterize the quantum spin structures, the analogue of the classical spin-flop configuration will be called 'spin-liquid' (SL) structure, and the analogue of the classical biconical configuration will be called supersolid (SS) structure, following the actual standard terminology.

We first briefly deal with the case $D / J=\Delta / 2[30,31]$. The ground state phase diagram in the $(\Delta, B / J)$ plane has been found $[30,31]$ to comprise AF, SL, SS, ferromagnetic (F), and (10), with a magnetization plateau at half saturation, phases. At small values of $\Delta$ and small fields, the Haldane phase is observed [30,34]. To compare, the corresponding classical spin chain shows a much broader biconical (BC) phase, being effectively replaced not only by the supersolid phase but also, largely, by the SL and (10) phases. The classical (10) phase becomes stable only in the limit of an Ising antiferromagnetic chain with a single-ion term, the Blume-Capel model [31].

Interesting information is given by the magnetization profiles, $m_{i}=\left\langle S_{i}^{z}\right\rangle$, with brackets, $\langle\ldots\rangle$, denoting quantum mechanical expectation values. In particular, we observe distinct profiles in the SS phase in between the AF and (10) and in between the AF and SL phases, respectively. For odd $L$, in the SS phase on approach to the (10) phase, the local magnetizations $m_{i}$ at odd sites stay close to one, while at even sites they tend roughly to zero. In contrast, in the SS phase on approach to the SL phase, the magnetizations on odd and even sites tend to take on the same values [31].

In the SL phase we find (studying situations with $\Delta$ exceeding $\approx 2.5$ ) two distinct types of profiles, for finite chains, when varying $M / L$, where $M$ is the total magnetization [31]: For $M<L / 2$, the profiles exhibit a broad plateau in the center of the chain, as expected for a classical spin-flop (SF) structure, while pronounced modulations in $m_{i}$ occur at $M>L / 2$, see figure 11 . This may signal a change from commensurate (C) to incommensurate (IC) structures [31], with an interesting quantum phase transition. The suggestion is confirmed and quantified by analysing the Fourier transform of the profiles, especially at $\Delta=3.5$. The modulation in the IC region of the SL phase is nicely described by the wavenumber (setting the lattice spacing equal to one) $q=2 \pi(1-m), m=M / L$. Such an IC modulation, with algebraic decay, is expected to hold in the entire SL phase of the spin-1/2 anisotropic Heisenberg chain in a field [35]. In the classical variant, we find no IC structures in the SF phase, for finite and infinite chains.

Let us now turn to the case of fixed exchange anisotropy, $\Delta=5.0$, varying the single-ion term, $D$. The ground state phase diagrams for the classical and the spin-1 chains are depicted in figures 12 and 13, using DMRG calculations for chains with up to 63 sites for the quantum case, and straightforward ground state considerations [25] (checked by Monte Carlo data) for the infinite 


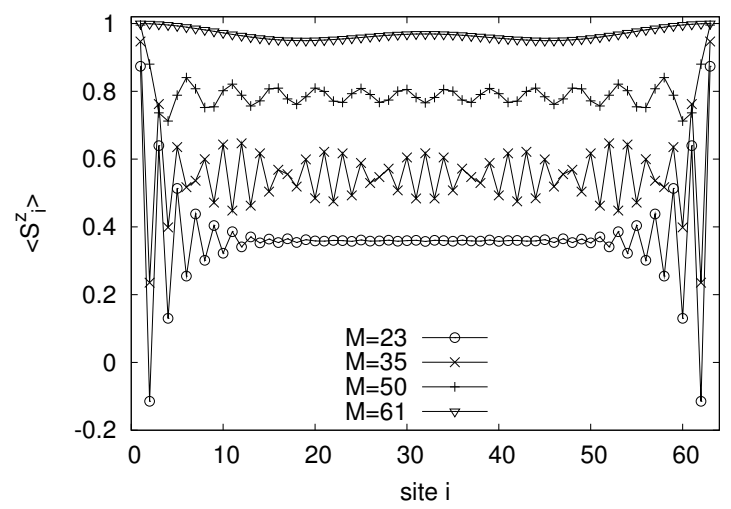

Figure 11. Magnetization profiles in the spin-liquid phase for $\Delta=2 D / J=3.5$ at various total magnetizations for the $S=1$ chain, equation (5), with $L=63$ sites.

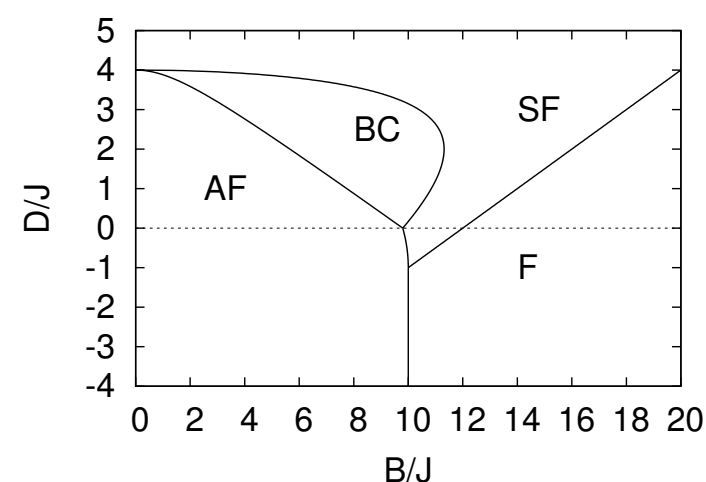

Figure 12. Ground state phase diagram of the classical infinite anisotropic Heisenberg chain, equation (5), with $\Delta=5.0$

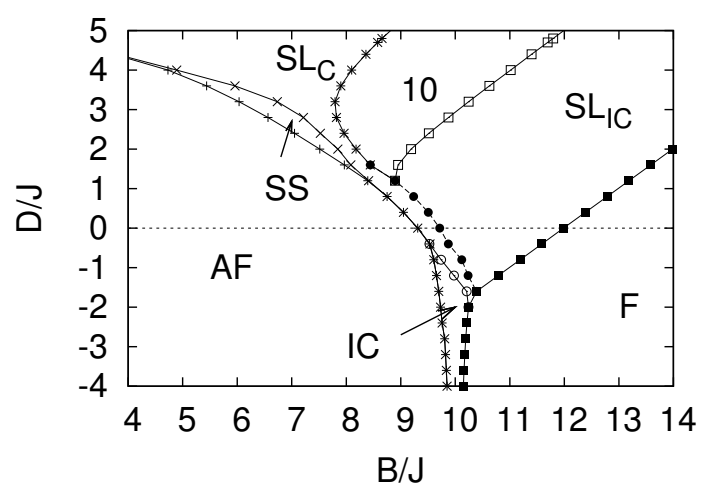

Figure 13. Ground state phase diagram of the spin-1 anisotropic Heisenberg chain, equation (5), with $\Delta=5.0$

classical chain. We considered positive and negative single-ion anisotropies, $D$, finding, especially, several intriguing quantum phase transitions.

For $D>0, \mathrm{BC}$ and SS phases are stable at zero temperature, see figures 12 and 13 . As in the case of $D / J=\Delta / 2$, the broad BC phase of the classical chain is effectively replaced, in the quantum chain, by the corresponding, rather narrow SS phase as well as SL and (10) phases.

In contrast to the case $D / J=\Delta / 2$, the supersolid phase is always bordered by the AF and SL phases. Accordingly, we observe only one type of magnetization profile. Illustrative examples are depicted in figure 14 , at $D / J=3.0$ and various fields. In the SS phase, at a given single-ion anisotropy, $D$, and field, $B$, the magnetization takes on different values at odd and even sites in the center of the chain. The local magnetization $m_{i}$ tends to acquire the same value at odd and even sites on approach to the SL phase in the quantum chain. Actually, as described above, the classical BC phase is characterized by two tilt angles $\Theta_{A}$ and $\Theta_{\mathrm{B}}$ for the two sublattices formed by neighboring sites, with the tilt angles approaching each other when getting closer to the SF phase $[15,25]$. Obviously, this behavior is completely analogous to the one depicted in figure 14 .

Increasing $D$, the 'large-D' phase [36-39] may eventually be stable, leading to other intriguing quantum phase transitions. It corresponds to the planar phase in the classical model with vanishing field, with the spin vectors pointing perpendicular to the $z$-axis, being the ground state for $D / J \geqslant 4$. The new phase may be expected to give rise to a SL phase at non-zero fields. A discussion of this interesting aspect is, however, beyond the scope of the present contribution. 


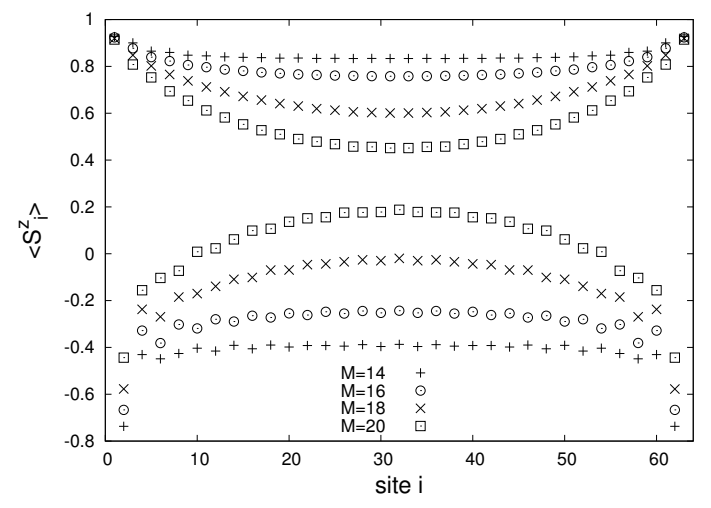

Figure 14. Magnetization profiles in the supersolid phase at $\Delta=5.0, D / J=3.0$, and fields $B / J$ (corresponding to total magnetizations $M$ ) between 6.5 and 6.8. Spin chains with $L=63$ sites are considered. The lower (upper) symbols denote the even (odd) sites.

For $D<0$, biconical or supersolid phases are no longer stable. In fact, only AF, SL (or SF), and $\mathrm{F}$ structures are encountered, in accordance with the discussion in the preceding subsection. The SL phase can be either commensurate, with a wide plateau in the magnetization profile away from the boundaries, or incommensurate, with modulations superimposed on the average magnetization. Obviously, there are two IC phases, see figure 13. The one, denoted by 'IC' in that figure, occurring essentially in between the AF and F phases, has been found before, having exponentially decaying transversal spin-spin correlations [29], in contrast to the usual spin-liquids with algebraic decay. It has no analogue in the classical model, see figure 12. The related transition between the IC and $\mathrm{SL}_{C}$ phases had been obtained before, being either of the first order or, at large average magnetization, continuous [29]. Our results agree with that description. We find another C-IC transition line between the $\mathrm{SL}_{C}$ and $\mathrm{SL}_{I C}$ phases at somewhat larger fields for a given $D / J$, see figure 12 (full circles). Note that this transition seems to take place at $M / L$ significantly larger than $1 / 2$ for $D<0$. Increasing $D, D>0$, in the vicinity of the (10) phase, the line goes over to the above discussed scenario with commensurate, $M / L<1 / 2$, and incommensurate, $M / L>1 / 2$, structures. By further increasing the planar single-ion anisotropy, $D / J$ being larger than roughly 3.6, we observe in the ' $\mathrm{SL}_{C}$ ' phase close to the (10) phase modulated structures for rather short chains, $L \leqslant 31$. The possible, additional C-IC border is not displayed in figure 13. Indeed, a more detailed analysis of that region, taking into account finite-size effects, is desirable.

\section{Summary}

In this contribution, we have presented the results of recent and current studies on a variety of anisotropic Heisenberg antiferromagnets in a field, elucidating the role of exchange and crystal field single-ion anisotropies, of lattice dimension, and of quantum effects.

The prototypical classical XXZ Heisenberg antiferromagnet with uniaxial exchange anisotropy in a field along the easy axis is shown to exhibit highly degenerate biconical ground states at the field separating $\mathrm{AF}$ and $\mathrm{SF}$ configurations. The $\mathrm{BC}$ structures give rise to a narrow disordered phase intervening between the $\mathrm{AF}$ and $\mathrm{SF}$ phases at low temperatures for square lattices. In contrast, for cubic lattices the $\mathrm{BC}$ fluctuations do not destroy the direct transition of the first order between the two ordered phases. Quantum effects seem to suppress the BC structures in the $S=1 / 2 \mathrm{XXZ}$ model on a square lattice, leading, presumably, to a transition of the first order between the AF and SF phases.

Thermally stable BC phases may occur when adding to the exchange anisotropy of the XXZ antiferromagnet, for instance, a quadratic or cubic single-ion anisotropy favoring a non-uniaxial ordering of the spins. The resulting phase diagrams for classical two- and three-dimensional magnets exhibit intriguing multicritical points. 
Studying ground state properties of $S=1$ anisotropic Heisenberg chains with an additional quadratic single-ion anisotropy, we observe, for finite chains, two distinct types of spin-liquid as well as two types of supersolid structures. The region of stability of the supersolid structures is substantially reduced, compared to that of the corresponding biconical structures in the classical case. Of course, there are no thermal phase transitions in one-dimensional magnets with short-range interactions. However, there are interesting quantum phase transitions at zero temperature.

\section{Acknowledgements}

We should like to thank, especially, A. Aharony, C. D. Batista, A. N. Bogdanov, T.-C. Dinh, R. Folk, A. Kolezhuk, N. Laflorencie, D. P. Landau, R. Leidl, P. Sengupta, and J. Sirker for useful discussions and information. A part of the research has been funded by the excellence initiative of the German federal and state governments.

\section{References}

1. Aharony A., J. Stat. Phys., 2003, 110, 659.

2. Shapira Y.- In: Multicritical Phenomena (Eds.: Pynn R., Skjeltorp A.) Plenum Press, New-York and London, 1983, p. 35.

3. Kosterlitz J.M., Nelson D.R., Fisher M.E., Phys. Rev. B, 1976, 13, 412.

4. Wissel Ch., Phys. Status Solidi B, 1972, 51, 669.

5. Bogdanov A.N., Zhuralev A.V., Rößler U.K., Phys. Rev. B, 2007, 75, 094425.

6. Selke W., Pokrovsky V.L., Büchner B., Kroll T., Eur. Phys. J. B, 2002, 30, 83.

7. Leidl R., Klingeler R., Büchner B., Holtschneider M, Selke W., Phys. Rev. B, 2006, 73, 224415.

8. Matsubara T., Matsuda H., Prog. Theor. Phys., 1956, 16, 569.

9. Matsuda T., Tsuneto T., Suppl. Prog. Theor. Phys., 1970, 46, 411.

10. Liu K.-S., Fisher M.E., J. Low Temp. Phys., 1973, 10, 655.

11. Nussinov Z., Physics, 2008, 1, 40.

12. Landau D.P., Binder K., Phys. Rev. B, 1981, 24, 1391.

13. Holtschneider M., Selke W., Leidl R., Phys. Rev. B, 2005, 72, 064443.

14. Zhou C., Landau D.P., Schulthess T.C., Phys. Rev. B, 2006, 74, 064407.

15. Holtschneider M., Wessel S., Selke W., Phys. Rev. B, 2007, 75, 224417.

16. Holtschneider M., Selke W., Eur. Phys. J. B, 2008, 62, 147.

17. Zhou C., Landau D.P., Schulthess T.C., Phys. Rev. B, 2007, 76, 024433.

18. Schmid G., Todo S., Troyer M., Dorneich A., Phys. Rev. Lett, 2002, 88, 167208.

19. Syljuåsen O.F., Sandvik A.W., Phys. Rev. E, 2002, 66, 046701.

20. Wessel S., Stoop N., Gull E., Trebst S., Troyer M., J. Stat. Mech-Theory and Experiment, 2007, $\mathrm{P} 12005$.

21. Landau D.P., Binder K., Phys. Rev. B, 1978, 17, 2328.

22. Bannasch G., Selke W., Eur. Phys. J. B, 2009, 69, 439.

23. Calabrese P., Pelissetto A., Vicari E., Phys. Rev. B, 2003, 67, 054505.

24. Folk R., Holovatch Yu., Moser G., Phys. Rev. E, 2008, 78, 041124.

25. Holtschneider M., Selke W., Phys. Rev. B, 2007, 76, 220405(R).

26. Bruce A.D., Aharony A., Phys. Rev. B, 1975, 11, 478.

27. Dinh T.-C., Folk R., Proc. Contributed Papers of Conf. on Statistical Physics: Modern Trends and Applications, Lviv, June 2009

28. Pelissetto A., Vicari E., Phys. Rep. 2002, 368, 549.

29. Tonegawa T., Okunishi K., Sakai T., Kaburagi M., Prog. Theor. Phys. Suppl., 2005, 159, 77.

30. Sengupta P., Batista C.D., Phys. Rev. Lett, 2007, 99, 217205.

31. Peters D., McCulloch I.P., Selke W., Phys. Rev. B, 2009, 79, 132406.

32. White S.R., Phys. Rev. B, 1993, 48, 10345.

33. Schollwöck U., Rev. Mod. Phys, 2005, 77, 259.

34. Haldane F.D.M., Phys. Rev. Lett, 1983, 50, 1153.

35. Kimura S., Takeuchi T., Okunishi K., Hagiwara M., He Z., Kindo K., Taniyama T., Itoh M., Phys. Rev. Lett., 2008, 100, 057202.

36. Schulz H.J., Phys. Rev. B, 1986, 34, 6372.

37. Mikeska H.-J., Kolezhuk A., Lect. Notes Phys. 2004, 645, 1. 
38. Chen W., Hida K., Sanctuary B.C., Phys. Rev. B, 2003, 67, 104401.

39. Albuquerque A.F., Hamer C.J., Oitmaa J., Phys. Rev. B, 2009, 79, 054412.

\title{
Класичні та квантові анізотропні Гайзенбергівські антиферомагнетики
}

\author{
В. Зельке ${ }^{1}$, Г. Баннаш ${ }^{2}$, М. Хольтшнайдер ${ }^{1}$, І.П. Маккаллок ${ }^{3}$, Д. Пітерс ${ }^{1}$, \\ С. Вессель ${ }^{4}$ \\ 1 Університет Аахена, Відділ теоретичної фізики, 52056 Аахен, Німеччина \\ 2 Інститут фізики комплексних систем, 01187 Дрезден, Німеччина \\ 3 Університет Квінсленда, Брісбейн, 4072, Австралія \\ 4 Університет Штутгарта, Інститут теоретичної фізики III, 70550 Штутгарт, Німеччина
}

Отримано 12 червня 2009 р., в остаточному вигляді - 30 червня 2009 р.

\begin{abstract}
Вивчаються класичні та квантові Гайзенбергівські антиферомагнетики з анізотропією обмінної взаємодії XXZ-типу та одно-іонними внесками від кристалічного поля квадратного і кубічного типу за полем. Ці магнетики проявляють велике різноманіття фаз, включаючи фазу з нахиленими спінами (або фазу "спінової рідини" у квантовому випадку) та двоконусну фазу (або надтекучу твердотільну фазу в рамках моделі квантового граткового газу, відповідно). В рамках формалізму основних станів, методів Монте-Карло та ренормалізаційної групи у підході матриці густини проаналізовано вплив квантових ефектів та розмірності гратки. Виявлено можливість появи цікавої критичної та мультикритичної поведінки при квантових та термоіндукованих фазових переходах.
\end{abstract}

Ключові слова: Гайзенбергівські антиферомагнетики, моделювання Монте-Карло, ренормгрупа у підході матриці густини, двоконусна фаза, надтекуча твердотільна фаза, мультикритична точка

PACS: $05.10 . \mathrm{Ln}, 75.10 . \mathrm{Jm}, 75.40 . \mathrm{Mg}, 75.40 . \mathrm{CX}$ 